\title{
ANALYTICAL BENDING ANALYSIS OF A CIRCULAR SANDWICH PLATE UNDER DISTRIBUTED LOAD
}

\author{
Hamed Vahdati Zadeh and Masoud Tahani \\ Department of Mechanical Engineering, \\ Ferdowsi University of Mashhad, Mashhad, Iran
}

\begin{abstract}
In this paper, bending analysis of a circular sandwich plate under distributed load with simply supported and clamped boundary conditions is investigated. First, the governing equations of the circular sandwich plate are obtained and they are solved using the Bessel functions. Then in order to validate the correctness of analytical results, numerical finite element method is used and its results are presented in the forms of contours and graphs. The results indicate that under distributed load, maximum deflection happens at 0.3 of outside radius, away from centre, and minimum deflection occurs at the outer edge of the circular sandwich plate. The results from analytical and numerical methods are compared and it shows that analytical method provides an acceptable accuracy.
\end{abstract}

\section{KEYWORDS}

Circular Sandwich Plate, Bending Analysis, Modeling, FEM, ABAQUS

\section{INTRODUCTION}

Circular and annular plates are popular in many engineering fields, including: mechanical engineering and medical equipment's engineering, and also they are classic subjects in the theory of elasticity due to their relatively simple geometry. Unlike composite plates, sandwich plates are usually composed of layers with quite different material properties and rigidities. Necessity of using the mentioned diversity in the material properties usually stems from the simultaneous different and in some circumstances, opposite design criteria that have to be met by the individual layers. Gupta et al. [1] investigated the axisymmetric bending of circular sandwich panel under traverse shear loading. Dynamic response of the circular sandwich plate was investigated under dynamic loadings in reference [2]. In this study, the numerical finite element method was used to verify the analytical relations. Ma and Wang $[3,4]$ studied the post-buckling and nonlinear bending behaviours of Functionally Graded Material circular plates based on classical plate theory and third-order plate theory. Evaluation of stress and strain in the cells of the human spine with the synthesized disk is a very complicated work and must be performed using numerical simulations [5]. Sepahi [6] investigated the effect of large deflections of the FGM circular plate under thermo-dynamic loading on a non-linear foundation using the square element. Saeedi et al. Investigated the axi-symmetric shear and buckling of a thick FGM circular plate using the third shear order theory [7]. Alipour M. M. and Shariyat M. studied the axisymmetric bending based on the elasticity equilibrium equations of Zigzag theory [8]. The non-linear analysis of FGM circular plate has been studied by Nosier A. And Fallah F. [9]. In this research the axis-symmetric 
traverse loading was applied according to the first shear deformation theory based on non-linear theory of Von-Karman.

In this paper, bending analysis of a circular sandwich plate under distributed load with simply supported and clamped boundary conditions is investigated. First, the governing differential equations are derived, and then are solved by means of Bessel function. ABAQUS software has been used for the numerical analysis.

\section{GOVERNING EQUATIONS}

A circular sandwich plate with the thickness of $2 h$, and the core radius of a. The thickness of the core and each of the shells is $2 h_{1}$ and $h_{2}$, as it is seen in Figure (1). Therefore we have: $h=h_{1}+h_{2}$.

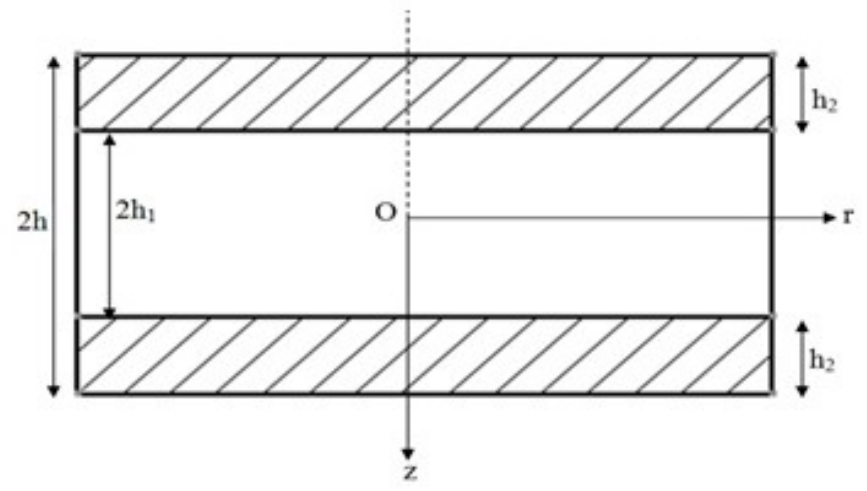

Figure 1. Cross-section of the circular sandwich panel

Regarding figure (1), the coordinates of the cylinder are: $r, \theta, z$, and in the mid-plane it is $\mathrm{z}=0$.

Two separators at the above and below surfaces are: $z= \pm h_{1}, \pm h$. The two surfaces have same materials, but the cores' materials are different. All the quantities in the core, upper surface, and the lower surface are shown by numbers 1 to 3 , respectively. Taking into account that it is assumed that bending of the circular plate happens in a non-symmetric manner, the displacement along $\theta$. Therefore, the displacement in the core and the shells in the cylindrical coordinates are as in relation (1):

$$
\begin{aligned}
& u_{1}(r, \theta, z)=z \psi_{1}(r, \theta) \\
& u_{2}(r, \theta, z)=-h_{1} \psi_{1}(r, \theta)+\left(z+h_{1}\right) \psi_{2}(r, \theta) \\
& u_{3}(r, \theta, z)=h_{1} \psi_{1}(r, \theta)+\left(z-h_{1}\right) \psi_{2}(r, \theta) \\
& v_{1}(r, \theta, z)=z \varphi_{1}(r, \theta) \\
& v_{2}(r, \theta, z)=-h_{1} \varphi_{1}(r, \theta)+\left(z+h_{1}\right) \varphi_{2}(r, \theta) \\
& v_{3}(r, \theta, z)=h_{1} \varphi_{1}(r, \theta)+\left(z-h_{1}\right) \varphi_{2}(r, \theta) \\
& w_{i}(r, \theta, z)=w(r, \theta)
\end{aligned}
$$

In the above equation we have $i=1,2,3$.

The total strain energy for an isotropic sandwich plate in the polar coordinate system is written as follows:

$$
V=\sum_{i=1}^{3} \int_{0}^{a} \int_{0}^{2 \pi} \int_{x_{1}}^{y_{1}}\left(\sigma_{r i} \varepsilon_{r i}+\sigma_{\theta i} \varepsilon_{\theta i}+\sigma_{\theta i i} \varepsilon_{\theta z i}+\sigma_{r \theta i} \varepsilon_{\theta r i}\right) r d z d \theta d r
$$


In the equation (2), the limits of the integration for $x_{i}$ to $y_{i}(i=1)$ is $-h_{1}$ to $h_{1}$, for $(i=2)-h$ to $-h_{1}$, and for $(i=3) h_{1}$ to $h$.

The strains results obtained from following equation:

$$
\left(N_{r i}, N_{\theta i}, M_{r i}, M_{\theta i}, Q_{r i}, Q_{\theta i}\right)=\int_{x_{i}}^{y_{i}}\left(\sigma_{r i}, \sigma_{\theta i}, z \sigma_{r i}, z \sigma_{\theta i}, z \sigma_{r \theta i}, k_{s} \sigma_{r z i}, k_{s} \sigma_{\theta z i}\right) d z
$$

variations of the total potential energy of the system, using the surface load of $p(r, \theta)$ is:

$U=-\int_{0}^{a} \int_{0}^{2 \pi} p(r, \theta) w r d \theta d r$

For a system in equilibrium, variations of the total potential energy $(T=U+V)$ would be insignificant with regards to the dependent parameters of $w, \psi_{1}, \psi_{2}, \varphi_{1}, \varphi_{2}$. In other words, we have:

$\delta T=\delta U+\delta V=0$

Equilibrium equations with the use of displacement term are achieved as follows:

$$
\begin{aligned}
& \frac{2 H_{1}}{3}\left(H_{1} G_{1}+3 H_{2} G_{2} G_{3}\right) \nabla_{1}^{2} \overline{\psi_{1}}+H_{2}^{2} G_{2} G_{3} \nabla_{1}^{2} \overline{\psi_{2}}+\frac{2}{3} H_{1}\left\{H_{1}\left(v_{1} G_{1}+1\right)\right\} \\
& +3 H_{2} G_{3}\left(v_{2} G_{2}+1\right) \frac{1}{R} \bar{\varphi}_{1, R \theta} \\
& +H_{2}^{2} G_{3}\left(v_{2} G_{2}+1\right) \frac{1}{R} \overline{\bar{\varphi}_{2, R \theta}}+\frac{2}{3} H_{1}\left(H_{1}+3 H_{2} G_{3}\right) \times \frac{1}{R^{2}} \overline{\psi_{1, \theta \theta}} \\
& H_{2}^{2} G_{3} \frac{1}{R^{2}} \overline{\psi_{2}, \theta \theta}-\frac{2}{3} H_{1}\left\{H_{1}\left(G_{1}+1\right)+3 H_{2} G_{3}\left(G_{2}+1\right)\right\} \frac{1}{R^{2}} \overline{\varphi_{1, \theta}} \\
& -H_{2}^{2} G_{3}\left(G_{2}+1\right) \times \frac{1}{R} \overline{\varphi_{2, \theta}}-2\left(\overline{\psi_{1}}+\overline{w_{, R}}\right)=0 \\
& H_{1} H_{2} G_{2} \nabla_{1}^{2} \overline{\psi_{1}}+\frac{2}{3} H_{2}^{2} G_{2} \nabla_{1}^{2} \overline{\psi_{2}}+\frac{2}{3} H_{2}^{2} \frac{1}{R^{2}} \overline{\psi_{2, \theta \theta}}+H_{1} H_{2} \frac{1}{R^{2}} \overline{\psi_{1, \theta \theta}} \\
& +H_{1} H_{2}\left(v_{2} G_{2}+1\right) \frac{1}{R} \bar{\varphi}_{1, R \theta}+\frac{2}{3} H_{2}^{2}\left(v_{2} G_{2}+1\right) \times \frac{1}{R} \overline{\varphi_{2, R \theta}}- \\
& H_{1} H_{2}\left(G_{2}+1\right) \frac{1}{R^{2}} \overline{\varphi_{1, \theta}}-\frac{2}{3} H_{2}^{2}\left(G_{2}+1\right) \times \frac{1}{R^{2}} \overline{\varphi_{2, \theta}}-2\left(\overline{\psi_{2}}+\overline{w_{, r}}\right)=0 \\
& \frac{2}{3} H_{1}\left(H_{1}+3 H_{2} G_{3}\right) \nabla_{1}^{2} \overline{\varphi_{1}}+H_{2}^{2} G_{3} \nabla_{1}^{2} \overline{\varphi_{2}}+\frac{2}{3} H_{1}\left(H_{1} G_{1}+3 H_{2} G_{2} G_{3}\right) \times \frac{1}{R^{2}} \overline{\varphi_{1, \theta \theta}} \\
& +H_{2}^{2} G_{2} G_{3} \frac{1}{R^{2}} \overline{\varphi_{2, \theta \theta}}+\frac{2}{3} H_{1}\left\{H_{1}\left(v_{1} G_{1}+1\right)+3 H_{2} G_{3}\left(v_{2} G_{2}+1\right)\right\} \frac{1}{R} \overline{\psi_{1, R \theta}} \\
& +H_{2}^{2} G_{3}\left(v_{2} G_{2}+1\right) \frac{1}{R} \overline{\psi_{2, R \theta}}+\frac{2}{3} H_{1}\left\{H_{1}\left(G_{1}+1\right)+3 H_{2} G_{3}\left(G_{2}+1\right)\right\} \times \frac{1}{R^{2}} \overline{\psi_{1, \theta}} \\
& +H_{2}^{2} G_{3}\left(G_{2}+1\right) \frac{1}{R^{2}} \overline{\psi_{2, \theta}}-2\left(\overline{\varphi_{1}}+\frac{1}{R} \overline{w_{, \theta}}\right)=0
\end{aligned}
$$


$H_{1} H_{2} \nabla_{1} \overline{\varphi_{1}}+\frac{2}{3} H_{2}^{2} \nabla_{1}^{2} \overline{\varphi_{2}}+H_{1} H_{2} G_{2} \frac{1}{R^{2}} \overline{\varphi_{1}}+\frac{2}{3} H_{2}^{2} G_{2} \frac{1}{R^{2}} \overline{\varphi_{2, \theta}}$

$+H_{1} H_{2}\left(v_{2} G_{2}+1\right) \frac{1}{R} \bar{\psi}_{1, R \theta}+\frac{2}{3} H_{2}^{2}\left(v_{2} G_{2}+1\right) \frac{1}{R} \bar{\psi}_{2, R \theta}$

$+H_{1} H_{2}\left(G_{2}+1\right) \frac{1}{R^{2}} \bar{\psi}_{1, \theta}+\frac{2}{3} H_{2}^{2}\left(G_{2}+1\right) \frac{1}{R^{2}} \bar{\psi}_{2, \theta}$

$-2\left(\bar{\varphi}_{2}+\frac{1}{R} \bar{w}_{, \theta}\right)=0$

$\left(H_{1}+H_{2} G_{3}\right)\left(\bar{w}_{, R R}+\frac{1}{R} \bar{w}_{, R}+\frac{1}{R^{2}} \bar{w}_{, \theta \theta}\right)+H_{1}\left(\bar{\psi}_{1, R}+\frac{1}{R} \bar{\psi}_{1}+\frac{1}{R}{\overline{\varphi_{1, \theta}}}_{1}\right)$

$+H_{2} G_{3}\left(\bar{\psi}_{2, R}+\frac{1}{R} \bar{\psi}_{2}+\frac{1}{R} \bar{\varphi}_{2, \theta}\right)+\frac{p(R, \theta)}{2 P}=0$

In the above equations:

$\nabla_{1}^{2}=\frac{d^{2}}{d R^{2}}+\frac{1}{R} \frac{d}{d R}-\frac{1}{R^{2}}$

$R=r / a, \mathrm{H}_{1}=h_{1} / a, \mathrm{H}_{2}=h_{2} / a$

$G_{1}=\lambda_{1} / \mu_{1}, G_{2}=\lambda_{2} / \mu_{2}, G_{3}=\mu_{2} / \mu_{1}$

$\overline{\psi_{1}}=\mu_{1} \psi_{1} / P, \overline{\psi_{2}}=\mu_{1} \psi_{2} / P, \bar{w}=\mu_{1} w / a P$

$\overline{\varphi_{1}}=\mu_{1} \varphi_{1} / P, \overline{\varphi_{2}}=\mu_{1} \varphi_{2} / P$

Also in the above $P$ is the average pressure on the surface. Regarding above equations, the following parameters could be defined:

$\overline{\psi_{1}}=\sum_{m=0}^{\infty}\left(S_{1 m, R}+\frac{m}{R} T_{1 m}\right) \cos m \theta$

$\overline{\psi_{2}}=\sum_{m=0}^{\infty}\left(S_{2 m, R}+\frac{m}{R} T_{2 m}\right) \cos m \theta$

$\overline{\varphi_{1}}=-\sum_{m=0}^{\infty}\left(\frac{m}{R} S_{1 m, R}+T_{1 m, R}\right) \sin m \theta$

$\overline{\varphi_{2}}=-\sum_{m=0}^{\infty}\left(\frac{m}{R} S_{2 m, R}+T_{2 m, R}\right) \sin m \theta$

$\bar{w}=\sum_{m=0}^{\infty} W_{m} \cos m \theta$

In the equations (8), $S_{1 m}, S_{2 m}, T_{1 m}, T_{2 m}$, and $W_{m}$ are a function of $R$. The load can also be definedas:

$$
p(R, \theta)=\sum_{m=0}^{\infty} p_{m}(R) \cos m \theta
$$

Using the above equations, the following equations achieved: 


$$
\begin{aligned}
& a_{9} \nabla^{4} T_{1 m}+a_{10}\left(\nabla^{2}-2\right) T_{2 m}=0 \\
& \nabla^{2}=\frac{d^{2}}{d R^{2}}+\frac{1}{R} \frac{d}{d R}-\frac{m^{2}}{R^{2}} \\
& a_{1}=2\left(H_{1}^{2} G_{1}+3 H_{1} H_{2} G_{2} G_{3}\right) / 3 ; a_{2}=H_{2}^{2} G_{2} G_{3}, a_{3}=H_{1} H_{2} G_{2}, \\
& a_{4}=2 H_{2}^{2} G_{2} / 3, a_{5}=H_{2} G_{2} G_{3}, a_{6}=H_{1}+a_{5}, a_{7}=2\left(H_{1}^{2} G_{1}+3 H_{1} H_{2} G_{3}\right) / 3 \\
& a_{8}=H_{2}^{2} G_{3}, a_{9}=H_{1} H_{2}, a_{10}=2 H_{2}^{2} / 3, \bar{p}_{m}(R)=p_{m}(R) / p
\end{aligned}
$$

The solution of equations (10) has two parts: Complementary Solution, and Particular Solution.

In the following equation $F_{m}$ replaces the three variables of $S_{1 m}, S_{2 m}$, and $W_{m}[1]$ :

$\left(b_{1} \nabla^{2}+b_{2}\right) \nabla^{4} F_{m}=0$

Therefore, the complementary solution is in a way that $A_{i m}, B_{i m}$, and $C_{i m}$ are the coefficient of integration.

$$
\begin{aligned}
& S_{1 m}=\left(A_{1 m}+A_{2 m} R^{2}\right) R^{m}+A_{3 m} J_{m}\left(n_{3} R\right) \\
& S_{2 m}=\left(B_{1 m}+B_{2 m} R^{2}\right) R^{m}+B_{3 m} J_{m}\left(n_{3} R\right) \\
& W_{m}=\left(C_{1 m}+C_{2 m} R^{2}\right) R^{m}+C_{3 m} J_{m}\left(n_{3} R\right)
\end{aligned}
$$

And the particular solution for the equations will be reached at as:

$$
\begin{aligned}
& S_{1 m}=\sum_{n}^{\infty} A_{1 m}^{n} J_{m}\left(K_{m n} R\right) \\
& S_{2 m}=\sum_{n}^{\infty} B_{1 m}^{n} J_{m}\left(K_{m n} R\right) \\
& W_{m}=\sum_{n}^{\infty} C_{m}^{n} J_{m}\left(K_{m n} R\right)
\end{aligned}
$$

By eliminating one of the variables, equation answer in term of Bessel functions is as follows:

$T_{1 m}=A_{4 m} J_{m}\left(R n_{1}\right)+A_{5 m} J_{m}\left(R n_{2}\right)$

$T_{2 m}=B_{4 m} J_{m}\left(R n_{1}\right)+B_{5 m} J_{m}\left(R n_{2}\right)$

Therefore, the final equations achieved as bellow:

$$
\begin{aligned}
& B_{4 m}=-a_{7}\left(2+n_{1}^{2}\right)^{A_{4 m}} /\left(a_{8} n_{1}^{2}\right) \\
& B_{5 m}=-a_{7}\left(2+n_{2}^{2}\right)^{A_{5 m}} /\left(a_{8} n_{2}^{2}\right)
\end{aligned}
$$

The load distribution over the sector in figure (2) is uniform, then in order to Calculate $D_{m}^{n}$ for $\alpha \neq 2 \pi$, equation (16) is resulted.

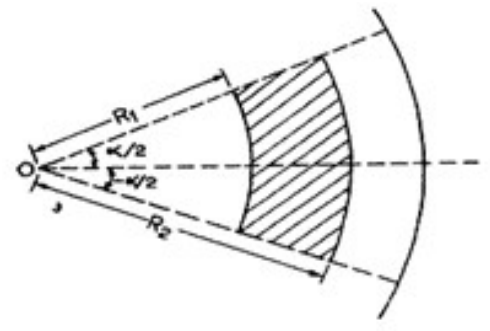

Figure 2. Choosing one point for solution of equations 


$$
\begin{aligned}
& \int_{0}^{1} R \bar{p}_{m}(R) J_{m}\left(K_{m n} R\right) d R=D{ }_{m}^{n} \int_{0}^{1} R J_{m}^{2}\left(K_{m n} R\right) d R=\frac{1}{2} D_{m}^{n}\left\{J_{m}^{\prime}\left(K_{m n}\right)\right\}^{2} \\
& \Rightarrow D_{m}^{n}=\frac{2}{\left\{J_{m}^{\prime}\left(K_{m n}\right)\right\}^{2}} \int_{0}^{1} R \bar{p}_{m}(R) J_{m}\left(K_{m n} R\right) d R
\end{aligned}
$$

$D_{m}^{n}$ obtained as follows:

$$
D_{m}^{n}=\frac{4 \sin (m \alpha / 2)}{m \alpha \bar{R} b\left\{J_{m}^{\prime}\left(K_{m n}\right)\right\}^{2}} \int_{R_{1}}^{R_{2}} R J_{m}\left(K_{m n} R\right) d R
$$

If the loading on the sector section is linear, then the coefficient of $D_{m}^{n}$ is calculated herein:

$$
D_{m}^{n}=\frac{24 \sin (m \alpha / 2)}{m \alpha b\left\{6 \bar{R}-2 \gamma\left(R_{1}^{2}+R_{2}^{2}+R_{1} R_{2}\right)\right\}\left\{J_{m}^{\prime}\left(K_{m n}\right)\right\}^{2}} \int_{R_{1}}^{R_{2}}(1-\gamma R) R J_{m}\left(K_{m n} R\right) d R
$$

\section{Modeling}

\subsection{Analytical model}

The schematic of the sandwich disk is presented in figure (3). The average load $\mathrm{P}$ and the dimensional values have been chosen as bellow.
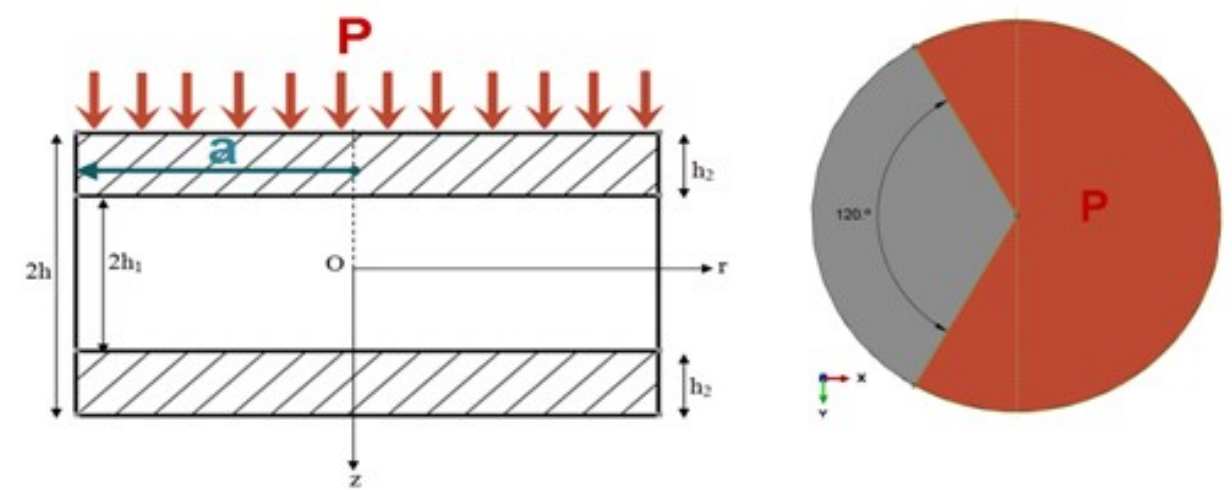

Figure 3. The schematic of the sandwich disk

$$
h_{1}=5 \mathrm{~mm}, \quad h_{2}=1 \mathrm{~mm}, \quad a=50 \mathrm{~mm}, \quad P=100 \mathrm{KN}, \alpha=240^{\circ}
$$

Table 1. Mechanical properties of different parts of the analytical model

\begin{tabular}{|c|c|c|}
\hline $\boldsymbol{V}$ & $\mathrm{E}(\mathrm{MPa})$ & Analytical model \\
\hline 0.3 & 5500 & (polyester resin/glass fibres) M450 Shells \\
\hline 0.41 & 896 & (polypropylene) Core \\
\hline
\end{tabular}

\subsection{Numerical model}

ABAQUS software is used for numerical analysis. Assuming that symmetrical loading, only half of the geometry of disk is modeled and symmetric boundary conditions is used for the shear plane. In following models 6063 S4R 4-linear node (with an approximate size of $1.5 \mathrm{~mm}$ for $100 \mathrm{~mm}$ disc diameter) is used. 


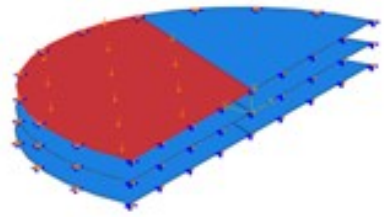

Figure 4. ABAQUS model and cross section of circular sandwich

\section{RESULTS}

\subsection{KN Pressure loading Analysis With Clamped boundary}

The results of analysis of static stress distribution for finite element model is presented in ABAQUS software. As can be seen with clamped conditions, for shell model, the maximum VonMises stress is $164 \mathrm{Mpa}$ and maximum deflection is $0.87 \mathrm{~mm}$.
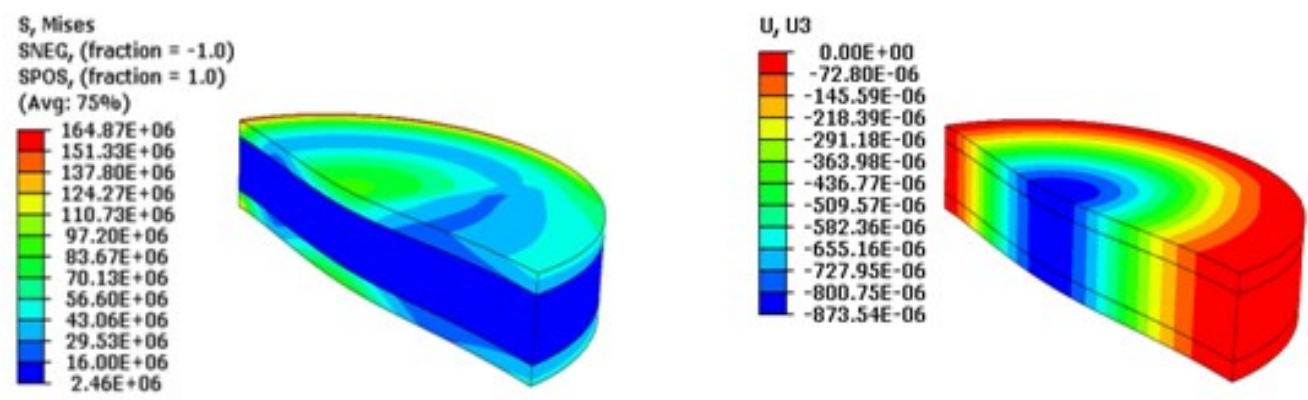

Figure 5. Stress and deflection distribution-pressure analysis under 100KN load with clamped boundry

\section{2. $100 \mathrm{KN}$ pressure loading analysis with simply supported boundary}

Regard to the results, with clamped boundary condition for shell model, maximum Von-Mises stress is $115 \mathrm{MPa}$ and maximum deflection is $0.89 \mathrm{~mm}$. As can be seen in figure (6), compared to the exact model with clamped boundary conditions, only the stress amount changed, but deflection is very similar.
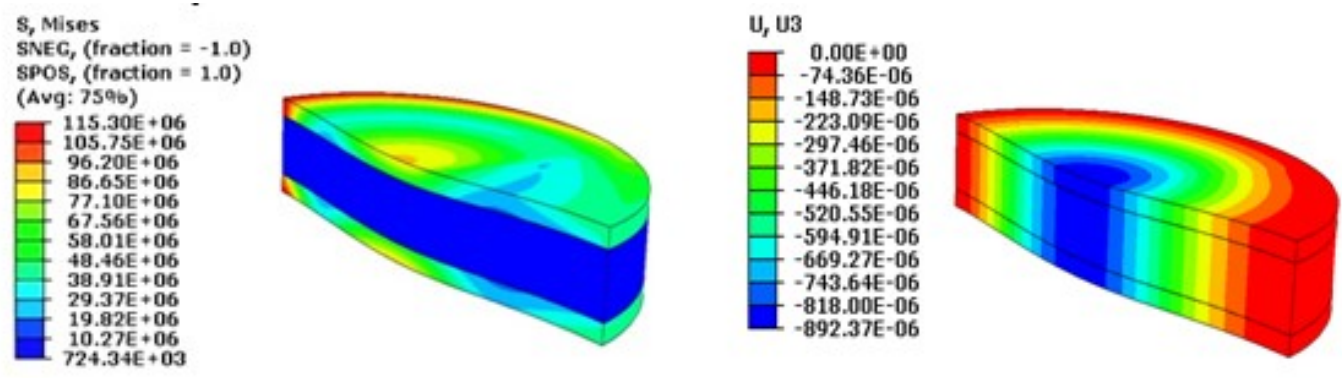

Figure 6. Stress and deflection distribution-pressure analysis under 100KN load with simply supported boundry

\subsection{Analytical method results}

Figure (7) shows the deflection distribution. Maximum deflection with value $1.3 \mathrm{~mm}$ occurs at $14 \mathrm{~mm}$ away from the center of the disk; whereas in finite element method of the shell model 
maximum deflection that happened at $10 \mathrm{~mm}$ away from the center was $0.89 \mathrm{~mm}$. Figure (7) that obtained by analytical approach, is showing decrease-increase trend in the deflection behavior.

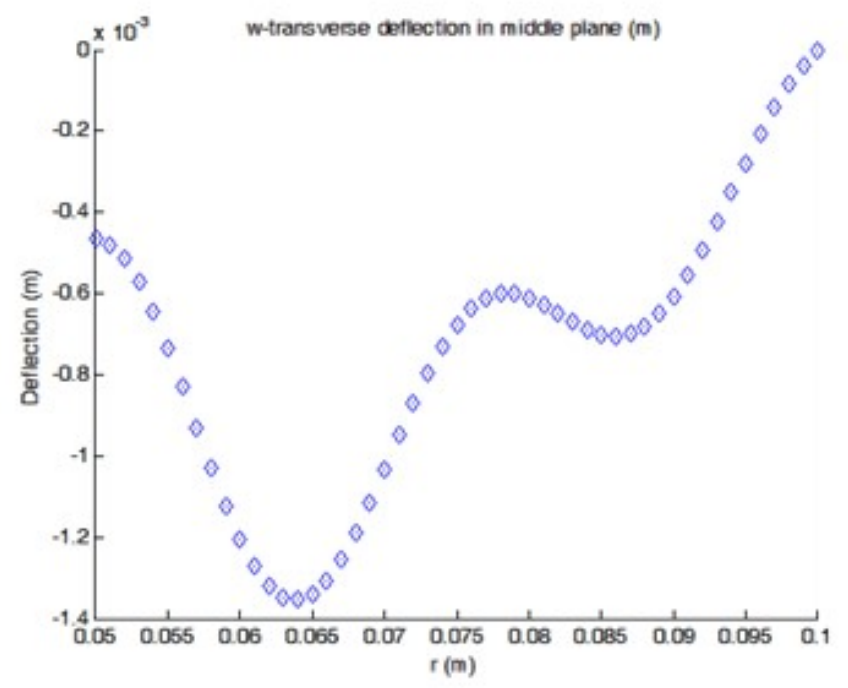

Figure 7. Results of analytical method in MATLAB software

\subsection{Comparison between the analytical and the numerical results}

Figure (8) displays deflection distribution at $(r=0$ to $50 \mathrm{~mm}, z=0, \theta=0)$ for two numerical analysis and analytical analysis with considering $100 \mathrm{KN}$ distributed load on the disk. As its shown, results from analytical method (MATLAB) has smaller error value than shell model and maximum deflection happens at $65 \mathrm{~mm}$ away from center, which means 0.3 of outer radius. Also the minimum deflection occurs at outer edge of the disk.

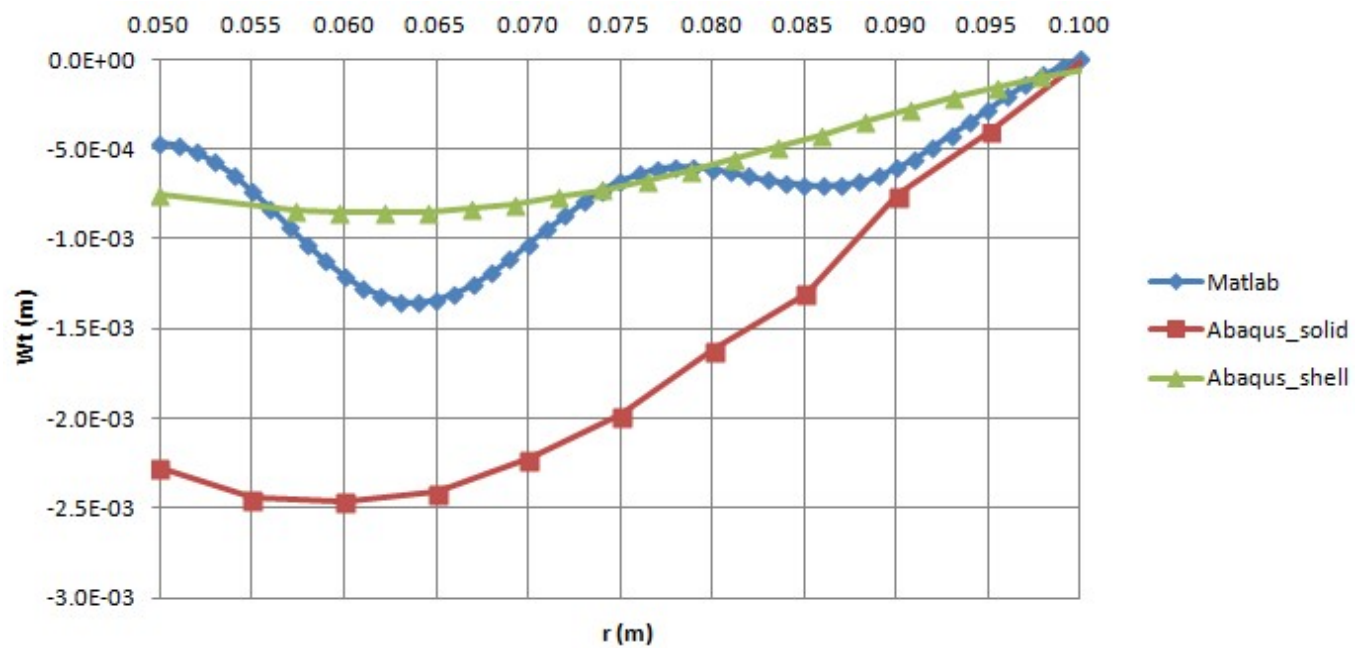

Figure 8. Comparison between analytical method result and results from numerical analysis from ABAQUS at $(r=0$ to $50 \mathrm{~mm}, z=0, \theta=0)$ 


\section{CONCLUSIONS}

In this study analytical method used to obtain deflection in circular sandwich plate with 3 layers and the results are compared with outcomes of the finite element method which is done by ABAQUS. According to the complexity of the equations in analytical method, only the deflection distribution along radius of the circular sandwich plate was obtained. Using of the Bessel functions, derivative and roots of them in expanding set of equations is the main source of error at analytical method which minimum error obtains in this method by increasing the number of the sentences in the Bessel function and convergence of the answer. Error in numerical method depends on the kind and number of elements and properties of the intersection. Stress distribution results for shell and solid models, from analysis of the bending composite disk, don't show a noticeable difference in a problem with different constraint type.

Also results for numerical method of shell model show that applying simply supported boundary and clamped boundary affect stress and deflection results for the presented problem in a way that replacing simply supported boundary with clamped boundary leads to smaller deflection and greater stress.

\section{REFERENCES}

[1] Anand P.Gupta, Kameshwar P.Sharma. (1982). Asymmetric bending of circular sandwich plate including transverse shear in facings, Indian J.Pure appl.Math., 13(12):1537-1550.

[2] V. S. Deshpande ,N. A. Fleck. (2004). Dynamic Response of a Clamped Circular Sandwich Plate Subject to Shock Loading, J. Appl. Mech 71(5), 637-645.

[3] L.S. Ma, T.J. Wang. (2003). Nonlinear bending and post-buckling of a functionally graded circular plate under mechanical and thermal loadings, Int. J. Solids Struct. 40, 3311-3330.

[4] L.S. Ma, T.J. Wang. (2004). Relationships between axisymmetric bending and buckling solutions of FGM circular plates based on third-order plate theory and classical plate theory, Int. J. Solids Struct. $111,85-101$.

[5] Bono.Ch., Garvin S.R. ( 2004). History and evolution of disc replacement, Spine J., 4, 145-150.

[6] Sepahi O., Forouzan M. R., Malekzadeh P. (2010). Large deflection analysis of thermo-mechanical loaded annular FGM plates on nonlinear elastic foundation via DQM. Composite Structures, 92: $2369-2378$.

[7] Saidi A.R, Rasouli A. ,Sahraee S. (2009). Axisymmetric bending and buckling analysis of FG circular plates using third order shear deformation theory. Composite Structures, 89:110-119

[8] Alipour MM, Shariyat M. (2012). An elasticity-equilibriumbased zigzag theory for axisymmetric bending and stress analysis of the functionally graded circular sandwich plates, using a Maclaurintype series solution. European Journal of Mechanics - A/Solids, 34: 78-101.

[9] Nosier A., Fallah F. (2009). Nonlinear analysis of functionally graded circular plates under asymmetric transverse loading. International Journal of Non-Linear Mechanics, 44: 928-942. 


\section{AUTHORS}

Hamed Vahdati Zadeh received his B.S. degree in Mechanical Engineering from Ferdowsi University of Mashhad, Iran, in 2011. He is currently studying his M.S. degree in Solid Mechanics. His main research interests include composite materials such as FGM and sandwich plates.

Masoud Tahani is currently a Professor and Head of the Department of Mechanical Engineering at Ferdowsi University of Mashhad, Iran. He received his B.S. in Mechanical Engineering from Ferdowsi University of Mashhad, Iran, in 1995. He then received his M.S.and Ph.D. degrees from Sharif University of Technology, Iran, in 1997 and 2003, respectively. Dr. Tahani's research interests include design of structures using advanced composites, mechanics of anisotropic materials, smart materials and structures,

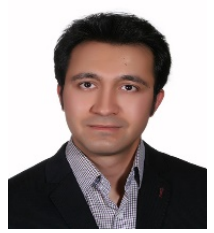
nanomechanics, and biomechanics. 